\title{
Myopia following penetrating keratoplasty for keratoconus
}

\author{
Stephen J Tuft, Fred W Fitzke, Roger J Buckley
}

\begin{abstract}
The frequent occurrence of spherical myopia after penetrating keratoplasty for keratoconus is partly the result of the excessive dioptric power of the grafted cornea which occurs when the diameter selected for the donor button is greater than the diameter of the host incision. This excessive power could be reduced by eliminating disparity between the diameters of the graft and host. To determine what proportion of the myopia in these eyes would persist as a result of axial myopia the axial lengths of 60 patients grafted for keratoconus and 25 emmetropic controls were compared. A keratometry, objective refraction, and contact probe ultrasonic biometry were performed on all eyes. A comparison of the results with a representational schematic eye indicated that the mean spherical refractive error of the grafted keratoconic eyes $(-4.83$ dioptres) was the combined effect of steepness of the corneal graft (mean radius of curvature $7.46 \mathrm{~mm}$ ) and an abnormally great axial length (mean $\mathbf{2 4 . 8 4} \mathrm{mm}$ ). The increased axial length was mainly the result of elongation of the posterior segment of the globe with a small contribution from an increased anterior chamber depth. Though axial myopia is common in keratoconus, a further study of $\mathbf{7 0}$ keratoconic eyes that had not been grafted showed no statistically significant correlation between the posterior segment length and the severity of corneal ectasia. These data suggest that even if excessive corneal power is eliminated after penetrating keratoplasty for keratoconus the associated axial myopia would still produce a mean spherical refractive error of at least $\mathbf{- 2 . 8}$ dioptres.

(Br F Ophthalmol 1992; 76: 642-645)
\end{abstract}

Penetrating keratoplasty is reserved for the minority of patients with keratoconus who cannot attain a satisfactory visual acuity with spectacles or contact lenses. Though a first graft to an eye is usually technically successful in terms of clarity and survival, ${ }^{1-3}$ astigmatism and residual myopia may prevent full visual rehabilitation. ${ }^{3-5}$ This has prompted interest in the origins of postoperative refractive error and methods for its reduction..$^{6-8}$

A disparity between the shape of the wound margins of the host and recipient is thought to be the primary cause for post-keratoplasty astigma- tism, ${ }^{9-11}$ but the origin of spherical myopia following keratoplasty for keratoconus is the subject of debate. It has been demonstrated that the use of an oversized donor corneal button produces a steeper graft contour which results in an excessive corneal dioptric power, ${ }^{6-812}$ but Wilson et al observed that grafted keratoconic eyes have a greater postoperative myopic spherical error than eyes grafted for Fuchs' dystrophy, even if the differences between diameters of the host and donor trephines $(0.25$ $\mathrm{mm})$ are the same in the two groups, ${ }^{8}$ and suggested a contribution by factors in addition to the graft contour toward the final refraction. The importance of the axial length of the globe was highlighted by Brooks et al who reported that the mean axial length of eyes with keratoconus was greater than emmetropic controls and that there is a correlation between the spherical myopic error following keratoplasty and the axial length of the eye. ${ }^{5}$

To characterise the origin of myopic error associated with keratoplasty for keratoconus we have compared the axial length and keratometry of grafted eyes and emmetropic control eyes. To assess if the grafted eyes could have been rendered emmetropic by eliminating overpower of the cornea we have used a representational schematic eye to determine the contribution of the axial length to the final refraction. To determine if there is a relationship between the severity of corneal disease in keratoconus and an elongation of the rest of the globe we studied the eyes of patients with keratoconus who had not undergone surgery.

\section{Patients and methods}

We examined the right eye of 60 phakic patients who had a clear penetrating keratoplasty for keratoconus and who had all sutures removed for at least 6 months. No eye had been grafted more than once. Patients were examined consecutively as they presented for review in clinic, but four eyes that had undergone refractive surgery for astigmatism were excluded from the study. Surgery had been performed by a number of different surgeons, but in the majority of cases the donor button was cut from the endothelial surface with a trephine 0.25 to $0.50 \mathrm{~mm}$ larger than the host and secured with a single 10/0 running suture. The follow-up period was defined as the interval between the removal of graft sutures and the observation point. The

Dr S Tuft.

Accepted for publication 21 May 1992 
Table 1 Clinical details of 60 patients who received a penetrating keratoplasty to their right eye

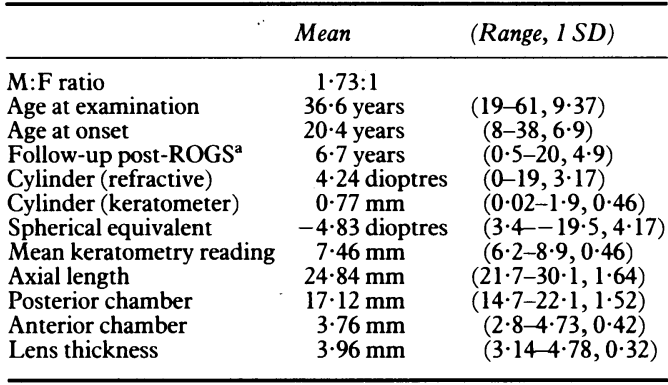

${ }^{a}$ Removal of graft sutures.

right eyes of 70 patients with keratoconus who had not had surgery were studied to compare their axial length and severity of their keratoconus. To establish our normal values for biometry and keratometry 25 volunteers were recruited from the hospital staff; they were age and sex matched with the unoperated keratoconus group and all had an unaided Snellen acuity of $6 / 6$.

Keratometry was performed using a Bausch and Lomb keratometer that could be modified
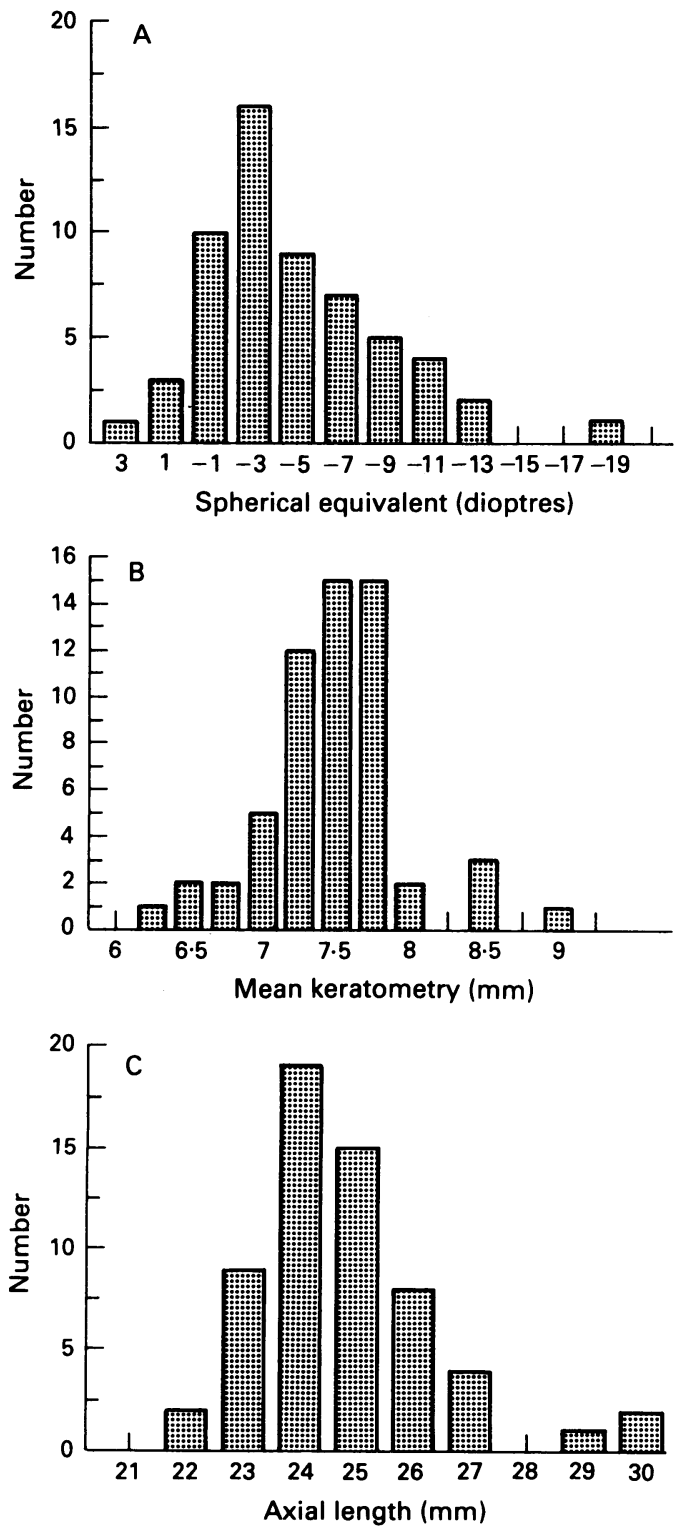

to measure high corneal powers. An objective refraction was obtained using a streak retinoscope without cycloplegia, and the axial length was measured along the visual axis using an ultrasonic contact probe (Allergan/Humphrey Biometer 820). Using this technique the anterior chamber depth is defined as the distance between the anterior surface of the cornea and the anterior surface of the lens, the lens thickness is the distance between the anterior and the posterior surfaces of the lens, and the posterior chamber depth is the distance between the posterior surface of the lens and the vitreoretinal interface. We have used the posterior chamber depth as an index of globe length because of the uncontrolled variables in the anterior segment such as corneal ectasia, indentation of the cornea by the contact probe, ${ }^{13}$ and alteration of the lens thickness with age and accommodation. We have estimated corneal flattening by the tip of the ultrasonic contact probe by comparing anterior chamber depth measurements from 10 emmetropic right eyes with readings obtained on the same eyes by an optical method (Haag Streit), taking five readings with each method from each eye. For comparison of the groups we have used the $t$ test, and for a comparison between readings we have used the paired $t$ test.

A computer model of the optics of the eye was used which emphasised an anatomically correct representation of the optical components and which allowed modification of each of the optical elements. ${ }^{14}$ is The surfaces were represented by aspherical, three-dimensional surfaces and the refractive index distribution was based on measured values from the literature. In particular, the crystalline lens was represented by a combination of graded refractive index changes within each of the major developmental zones along with a step change in refractive index corresponding to the discontinuities visible in slit-lamp images. The retinal surface was represented as aspherical and based on measured values. The parameters of corneal curvature and axial position of the surfaces were modified to incorporate the measured values from this study and the resulting optical effects computed. We have assumed that the refractive indices of the lens components are the same in the three study groups.

\section{Results}

All 60 grafted right eyes in this study had a corrected Snellen acuity of 6/9 or better. Contact lenses were the preferred method of visual correction for $55 \%$ of patients at the time of observation, $35 \%$ wore spectacles, and $10 \%$ wore no correction. The characteristics of the 60 patients are presented in Table 1. From the distribution of the spherical equivalents (Fig 1A), it may be seen that there is a skew toward a myopic error and only two eyes were more than 0.25 dioptres hypermetropic. The distribution of the mean keratometric readings (Fig 1B) indicates a skew toward steeper corneas following keratoplasty, with a mean value of $7.46 \mathrm{~mm}$ compared with $7 \cdot 80$ for emmetropic control eyes. The distribution of the axial lengths is skewed towards longer eyes (Fig 1C). 


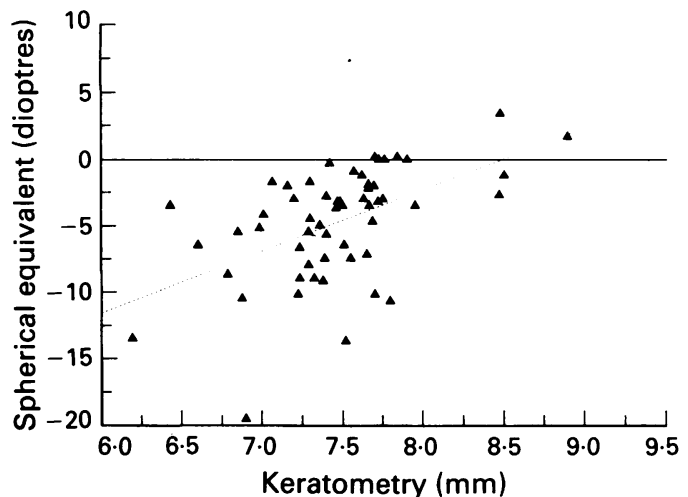

Figure 2 Scatter plot of spherical refraction of 60 grafted right eyes versus their mean keratometry. There is a significant tendency for eyes with steeper corneas to have a greater myopic error.

When we compared the final spherical refraction with the mean keratometry (Fig 2), the posterior chamber depth, or the axial length (Fig 3) there was a positive correlation for keratometry (correlation coefficient $0.529, \mathrm{p}<0.001$ ) and axial length (correlation coefficient -0.738 , $\mathrm{p}<0.001$ ), such that a final myopic error was associated both with steeper corneas and an increased globe length. However, when we compared the posterior chamber depth with the mean keratometry there was no correlation (correlation coefficient $0.0335, \mathrm{p}=0.8$ ), indicating that steeper corneal grafts did not tend to occur in longer eyes.

A comparison between the mean keratometry and the posterior chamber depth of keratoconus eyes that had not had a penetrating keratoplasty showed no significant correlation (Fig 4) (correlation coefficient $0.0676, \mathrm{p}=0.596$ ) which suggests that there is not a direct relationship between the severity of keratoconus, as indicated by ectasia of the cornea, and ectasia of the rest of the globe. There was also no statistically significant difference between the axial lengths or posterior chamber depths of the groups of keratoconic eyes that had received a penetrating graft and those that had not (Table 2); however both groups of keratoconic eyes were significantly longer than emmetropic controls $(\mathrm{p}<0.001)$. We confirmed a significant $(\mathrm{p}<0.001)$

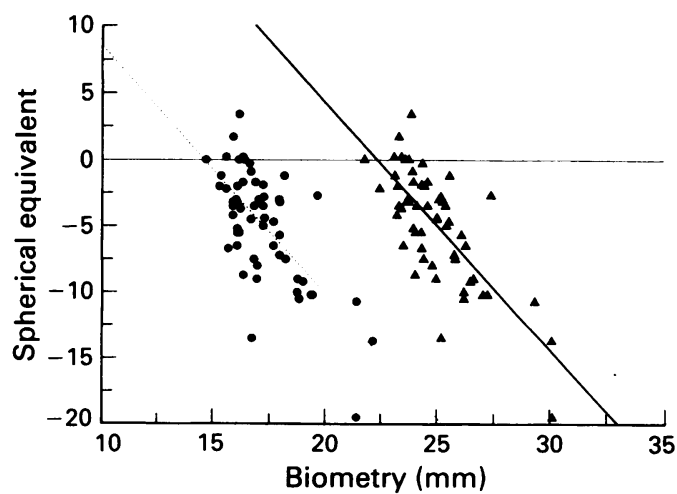

Figure 3 Scatter plots of the spherical equivalent of grafted eyes versus both their posterior chamber depth (circles) and axial length (triangles) measured by A-scan. There is a significant tendency for greater myopia to occur in eye with greater axial lengths $(p=0.001)$ and with greater posterior chamber depths $(p=0 \cdot 001)$. These data exclude an effect solely due to corneal ectasia as a result of the steep graft.

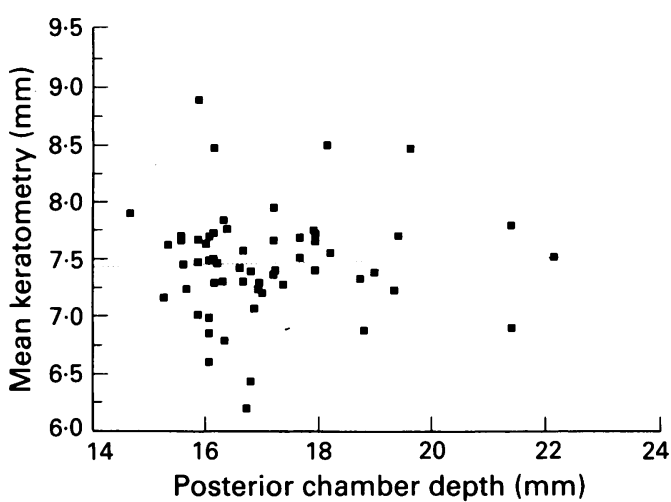

Figure 4 Scatter plot of the mean keratometry of eyes with keratoconus that had not been grafted versus their posterior chamber length. There is no correlation between severity of disease (steepness of the cone) and elongation of the eye.

difference in the measurement for the anterior chamber depth of the normal eye when we compared an optical method (mean 3.57 (SD 0.32 ) $\mathrm{mm}$ ) with an ultrasonic contact probe (mean $3 \cdot 17$ (SD 0.29) $\mathrm{mm}$ ), which would produce low values for axial length measurements with the latter method.

The contribution of the difference in the grafted corneal curvature from the normal was calculated to be $-1 \cdot 2$ dioptres. The contribution from the increased axial length was $-2 \cdot 8$ dioptres using standard values $(24.0 \mathrm{~mm})^{1+15}$ or $-4 \cdot 6$ dioptres using data uncorrected for corneal indentation. In total, these two components were calculated to contribute between $-4 \cdot 0$ to $-5 \cdot 8$ dioptres of refractive error. The contribution to the final refractive error from axial length was therefore approximately three times that of the corneal curvature contribution. The contribution of lens thickness differences and of the position of the lens relative to the other optical components was calculated to contribute less than $0 \cdot 1$ dioptres each.

\section{Discussion}

The objective of penetrating keratoplasty for keratoconus is both to flatten the radius of curvature of the anterior corneal surface and to remove axial corneal scarring. An emmetropic eye is an ideal end-point, but corneal astigmatism and residual spherical myopia usually occur. ${ }^{4516}$ Spherical myopia has been attributed to excessive corneal power owing, in part, to the use of an oversize donor button..$^{6-817}$ The practice of using an oversized button for keratoplasty was

Table 2 Ocular biometry-right eyes

\begin{tabular}{|c|c|c|c|}
\hline & Number & Mean & $S D$ \\
\hline $\begin{array}{l}\text { Axial length } \\
\text { Normals } \\
\text { Keratoconus - grafted } \\
\text { Keratoconus - ungrafted } \\
\text { Comparing normal and gr } \\
\text { Comparing grafted with u }\end{array}$ & $\begin{array}{l}25 \\
60 \\
70 \\
\text { yes with k } \\
\text { ed eyes wit }\end{array}$ & $\begin{array}{c}23 \cdot 46 \\
24 \cdot 84 \\
24 \cdot 47 \\
\text { toconus } \\
\text { eratoco }\end{array}$ & $\begin{array}{r}0.78 \\
1.65 \\
1.65 \\
\times 0.00 \\
=0.2\end{array}$ \\
\hline $\begin{array}{l}\text { Posterior chamber length } \\
\text { Normals } \\
\text { Keratoconus - grafted } \\
\text { Keratoconus - ungrafted } \\
\text { Comparing normal and gr } \\
\text { Comparing grafted with u }\end{array}$ & $\begin{array}{l}25 \\
60 \\
70 \\
\text { yes with k } \\
\text { ed eyes wit }\end{array}$ & $\begin{array}{l}16 \cdot 42 \\
17 \cdot 12 \\
17 \cdot 05 \\
\text { toconus } \\
\text { eratoco }\end{array}$ & $\begin{array}{r}0.72 \\
1.52 \\
1.52 \\
\times 0.00 \\
=0.7\end{array}$ \\
\hline
\end{tabular}


introduced to reduce the incidence of wound leaks and aphakic glaucoma in the early postoperative period ${ }^{18}$; however glaucoma is not associated with keratoplasty in phakic patients. ${ }^{19-23}$ A $0.2 \mathrm{~mm}$ difference between the radius of the donor button and host bed has therefore been recommended to safely minimise wound disparity. ${ }^{24-26} \mathrm{~A}$ further reduction in spherical myopia was reported by Girard et al who used a donor button smaller than the recipient. ${ }^{12}$

Although the effect of excessive power of the cornea may have been exaggerated in this series by the use of some donor buttons $0.5 \mathrm{~mm}$ larger than the host trephine, our results indicate that the greater proportion of the spherical myopia is the result of an abnormally large axial length. Even by eliminating excessive corneal power approximately $80 \%$ of the study group would still be myopic. Unfortunately penetrating keratoplasty reduces the central corneal ectasia of keratoconus but has little effect in reducing the axial length of the globe.

We have considered whether it may be possible to modify the final refraction by altering the relative sizes of the donor and host trephine - for example, attempting to obtain a flatter graft in an eye with a high axial length, but consider that the safety and benefit of using donor buttons cut smaller than the donor trephine is as yet unproved. In practical terms the individual variability of the final corneal contour and the gradual steepening of the cornea in the postoperative period ${ }^{27}$ may make it difficult to predict accurately the final refraction. It may also be argued that as long as patients continue to require contact lenses to overcome corneal astigmatism the presence of a coexisting myopia is of little significance.

Finally, it has been established that there is an association between keratoconus and simple myopia, and it has even been suggested that the two conditions may share a common disorder of connective tissue metabolism. ${ }^{28}$ However, unlike Brookes $e t a l,{ }^{5}$ when we used either keratometry or the need for penetrating keratoplasty as criteria for the severity of keratoconus, we were unable to establish a direct relationship between the length of the posterior segment of keratoconic eyes and the severity of their corneal disease.

Since acceptance of the paper the authors acknowledge the publication of a paper by Lanier et al who obtained similar values graphy. They measured a mean reduction in globe length of
$0.91 \mathrm{~mm}$ in eyes receiving a penetrating keratoplasty with a donor trephine $0.3 \mathrm{~mm}$ smaller than the host.

Lanier JD, Bullington RH, Prager TC. Axial length in keratoconus. Cornea 1992; 11: 250-4.

1 Paglen PG, Fine M, Abbott RL, Webster RG. The prognosis for keratoplasty in keratoconus. Ophthalmology 1982; 89: $651-4$

2 Epstein RJ, Seedor JA, Dreizen NG, Stulting RD, Waring GO, Wilson LA, et al. Penetrating keratoplasty for herpes simplex keratitis and keratoconus. Allograft rejection and survival. Ophthalmology 1987; 94: 935-44.

3 Troutman RC, Lawless MA. Penetrating keratoplasty for keratoconus. Cornea 1987; 4: 298-305.

4 Troutman RC, Meltzer M. Astigmatism and myopia in keratoconus. Trans Am Ophthalmol Soc 1972; 70: 265-77.

5 Brooks AMV, Robertson IF, Mahoney A-M. Ocular rigidity and intraocular pressure in keratoconus. Aust $\mathcal{f}$ Ophthalmol and intraocular p

6 Perry HD, Foulks GN. Oversize donor buttons in corneal transplantation surgery for keratoconus. Ophthalmic Surg 1987; 18: 751-2.

7 Duran JA, Malvar A, Diez E. Corneal dioptric power after penetrating keratoplasty. Brf Ophthalmol 1989; 73: 657-60.

8 Wilson SE, Bourne WM. Effect of recipient-donor trephine size disparity on refractive error in keratoconus. Ophthalmology 1989; 96: 299-305.

9 Olson RJ. The effect of scleral fixation ring placement and trephine tilting on keratoplasty wound size and donor shape. Ophthalmic Surg 1981;12: 23-6.

10 Cohen KL, Tripoli NK, Pellom AC, Kupper LL, Fryczkowski AW. Effects of tissue fit on corneal shape after transplantation. Invest Ophthalmol Vis Sci 1984; 25: 1226-31.

11 Cohen KL, Holman RE, Tripoli NK, Kupper LL. Effect of trephine tilt on corneal button dimensions. Am f Ophthalmol 1986; 101: 722-5.

12 Girard LJ, Eguez I, Esnaola N, Barnett L, Maghraby A. Effect of penetrating keratoplasty using grafts of various sizes on keratoconic myopia and astigmatism. F Cataract Refract Surg 1988; 14: 541-7.

13 Snead MP, Rubinstein MP, Hardman Lea S, Haworth SM. Calculated versus A-scan result for axial length using different types of ultrasound probe tip. Eye 1990; 4: 718-22.

14 Fitzke FW. Optical properties of the eye. Invest Ophthalmol Vis Sci (Supp) 1981; 20: 144.

15 Fitzke FW, Hayes BP, Hodos W, Holden AL. Electrophysiological optometry using Scheiner's principle in the pigeon eye. F Physiol 1986; 369: 17-31.

16 Jensen AD, Maumenee AE. Refractive errors following keratoplasty. Trans Am Ophthalmol Soc 1974; 72: 123-31.

17 Perl T, Charlton KH, Binder PS. Disparate diameter grafting: astigmatism, intraocular pressure, and visual acuity. Ophthalmology 1981; 88: 774-81.

18 Foulks GN, Perry HD, Dohlman CH. Oversize corneal donor grafts in penetrating keratoplasty. Ophthalmology 1979; 86: $490-4$

19 Irvine AR, Kaufman HE. Intraocular pressure following penetrating keratoplasty. Am $\mathcal{F}$ Ophthalmol 1969; 68: 83544.

20 Zimmerman T, Olson R, Waltman S, Kaufman H. Transplant size and elevated intraocular pressure postkeratoplasty. Arch Ophthalmol 1978; 96: 2231-3.

21 Olson RJ. Aphakic keratoplasty: determining donor size to avoid elevated intraocular pressure. Arch Ophthalmol 1978; 96: 2274-6.

22 Heidemann DG, Sugar A, Meyer RF, Musch DC. Oversized donor grafts in penetrating keratoplasty: a randomized trial. Arch Ophthalmol 1985; 103: 1807-11.

23 Polac FM. Glaucoma and keratoplasty. Cornea 1988; 7: 67-70. 4 Olson RJ. Variation in corneal graft size related to trephine technique. Arch Ophthalmol 1979; 97: 1323-5.

25 Troutman RC. Astigmatic considerations in corneal graft. Ophthalmic Surg 1979; 10: 21-6.

26 Van Rij G, Waring GO III. Configuration of corneal trephine opening using five different trephines in human donor eyes. opening using five different trephines

27 Ruben M, Colebrook E. Keratoconus, keratoplasty curvatures and contact lens wear. Brf Ophthalmol 1979; 63: 268-73.

28 Ihalainen A. Clinical and epidemiological features of keratoconus. Genetic and external factors in the pathogenesis of the disease. Acta Ophthalmol (Kbh) 1986; 64: 5-64. 